\title{
¿Qué ideas sobre su derecho a la intimidad en la escuela construyen niños y niñas?'
}

\author{
Axel César Horn² \\ https://orcid.org/0000-0003-1618-0539
}

\section{Resumen}

Se presentan las ideas infantiles de los niños y las niñas sobre el derecho a la intimidad en la escuela, surgidas de una investigación realizada en la Ciudad de Buenos Aires de la República Argentina. Los y las niñas estudiados/as tienen entre 7 y 12 años. El artículo muestra cuales son los grupos de ideas elaborados por estos sujetos. En la investigación se utilizó como estrategia metodológica entrevistas clínico-críticas a partir de la presentación de narrativas en las que se planteaba la vulneración del derecho a la intimidad en la escuela. Las ideas encontradas se dividen en tres grandes grupos: un grupo de sujetos no reconoce el derecho, otro lo reconoce, pero limitado en aquellas situaciones en las que dicho derecho tensiona alguna característica escolar, y un grupo menor de sujetos reconoce al derecho independiente de las características de la escuela. El artículo postula que la construcción de las ideas infantiles sobre el derecho a la intimidad se produce por una trabajosa labor intelectual mientras participan en contextos institucionales que no siempre son cuidadosos de la información personal de los y las niñas. Los resultados expuestos posibilitan una discusión acerca de las formas de construcción situada de nociones sociales por parte de los sujetos y las características que asume el desarrollo desde una perspectiva psicológica constructivista que pone en el centro el contexto social en el que los sujetos participan. Las preguntas que ordenan la discusión son las siguientes: ¿Existe desarrollo conceptual en este tipo de ideas sociales? De existir ¿En qué términos puede ser planteado?

\section{Palabras clave}

Derecho a la intimidad - Escuela - Conocimiento social - Desarrollo cognoscitivo.

1- El artículo se enmarca en el Proyecto de investigación UBACYT 2018-2021 20020170100222BA: Restricciones a los procesos de construcción conceptual en el dominio de conocimiento social: posibilidades y obstáculos para el programa de investigación constructivista. Director: Dr. José Antonio Castorina; Codirectora: Dra. Alicia Barreiro. Universidad de Buenos Aires.

2- Universidad Nacional de La Plata, La Plata, Argentina. Contacto: axelcesarhorn@gmail.com. 


\section{What ideas do boys and girls construct about their right to privacy at school? *}

\section{Abstract}

This paper presents the ideas that boys and girls have about the right to privacy at school, emerging from research conducted in the city of Buenos Aires, Argentina. The boys and girls studied are between the ages of 7 and 12. The article shows which group of ideas were developed by these subjects. In this research, clinical-critical interviews were used as a methodological strategy, based on the presentation of narratives addressing the violation of the right to privacy in schools. The resulting ideas are divided into three main groups: one group of subjects does not recognize this right, another recognizes it, although limited to those situations where this right strains some school characteristic, and a smaller group of subjects recognizes the right independently of the characteristics of the school. The article postulates that the construction of childhood ideas about the right to privacy occurs through hard intellectual work while participating in institutional contexts that are not always careful of children's personal information. The results exposed facilitate the discussion on the situated construction of social notions by the subjects and the characteristics assumed by development from a psychological constructivist perspective that puts the social context in which the subjects participate at the center. The questions ordering the discussion are as follows: Does conceptual development exist in this type of social ideas? If so, in what terms can it be raised?

\section{Keywords}

Right to privacy - School - Social knowledge - Knowledge development.

\section{Introducción}

En el campo de la psicología del desarrollo se han realizado investigaciones sobre la adquisición de nociones sociales (KARMILOFF-SMITH, 1994; HIRSCHFEL, 2002; CASTORINA; FAIGENBAUM, 2000; CASTORINA; LENZI; FERNÁNDEZ, 2000). Entre ellas no abundan las investigaciones que tengan como objetivo describir la construcción conceptual infantil sobre derechos específicos y es menor aún la cantidad de estudios que se refieren a las ideas que niños y niñas elaboran sobre el derecho a la intimidad.

Esta vacancia tiene algunas excepciones en el campo específico de la psicología de inspiración piagetiana donde se han realizado indagaciones sobre las ideas infantiles acerca de la privacidad, la intimidad, el secreto y a sus derechos (HELMAN; CASTORINA, 2005, 2007; HELMAN, 2010; HORN; CASTORINA, 2010; FERREYRA; HORN; CASTORINA, 2012, 2018; LA TAILLE; BEDOIA; GIMÉNEZ, 1991; LA TAILLE, 1995, 1996, 1998). 
Sin embargo, lejos de ser un conjunto de estudios compacto y sin divergencias sobre un tema común, abundan las divergencias teóricas y metodológicas. Desde algunos -Helman y Castorina (2005, 2007), Helman (2010), Horn y Castorina (2010), Ferreyra, Horn y Castorina (2012)- se afirma que la construcción conceptual de nociones sobre el derecho es inseparable de la participación del sujeto en contextos en los que el derecho o la noción tiene un determinado lugar y cumplimiento. En cambio, otros investigadores, como La Taille $(1995,1996)$, sugieren que este tipo de nociones los niños y las niñas las elaboran sin tener en cuenta las condiciones sociales en las que el sujeto participa. De esta forma se sostiene un proceso cognoscitivo individual que se realizaría en soledad.

Este artículo se propone, en primer lugar, presentar los resultados de una investigación donde se describe el desarrollo de las conceptualizaciones infantiles sobre el derecho a la intimidad en la escuela. En segundo lugar, plantear una discusión en torno al concepto de desarrollo. Más específicamente ¿puede seguir sosteniéndose la noción de desarrollo de nociones sociales que están fuertemente vinculadas con la participación del niño en contextos específicos? Y si se sostiene, en qué condiciones puede hacerse.

\section{Investigaciones de las ideas infantiles sobre la intimidad y su derecho}

El investigador Yves de La Taille (1991, 1995, 1996; BATAGLIA; FERREIRA; SALADINI, 2020) ha estudiado la génesis de la noción de secreto y su derecho y otros fenómenos morales, así como la construcción de la frontera moral de la intimidad.

Desde la perspectiva del autor, la consolidación de estas nociones forma parte de la constitución del psiquismo. La posibilidad de establecer que algunos aspectos de la vida personal serán considerados secretos implica reconocer un espacio privado ajeno a la intervención de los otros. En esta dirección, interpreta la construcción de la frontera de la intimidad, siguiendo a Altman (1979), en los términos de una "[...] frontera entre uno y los otros que garantiza los límites del proceso de interacción social” (LA TAILLE; BEDOIA; GIMÉNEZ, 1991, p. 92). Es discutible la tesis del autor que sostiene a la frontera íntima como un hecho universal, sin considerar su historicidad. Más aun, según su propuesta esta frontera se alcanza como resultado de la naturaleza humana:

[...] la privacidad parece corresponder a una necesidad humana, y si es cierto que sus formas varían en las diferentes épocas y culturas, también es verdad que su presencia es siempre identificable. (LA TAILLE, 1998, p. 137).

Es de particular interés para nosotros el estudio de La Taille, Bedoia y Giménez (1991) sobre la génesis del derecho al secreto. Esta investigación encuentra que desde los cuatro años los niños y las niñas reconocen que pueden tener secretos, aunque no consideran esto como un derecho. Este es construido posteriormente: los niños y niñas de ocho años pueden reconocer su derecho a no hacer pública una determinada información personal cuando es solicitada por otro niño, pero solo puede sostener tal derecho ante 
los adultos a los 12 o 13 años, aunque consideran que es más conveniente dar esta información a los adultos si la requieren.

¿Cómo explica el autor esta evolución de la noción de derecho al secreto? Se inspira en la teoría piagetiana y sostiene que la consolidación de la idea de derecho a los ocho años se debe, a nivel individual, a la construcción de la reversibilidad operatoria que le permite establecer una autonomía intelectual y, a nivel social, es el momento del descentramiento de las acciones del yo y la coordinación con las acciones y comunicaciones de los otros. Piaget (1971) llamó a esta relación social cooperación postulando que la misma conduce a la construcción de una autonomía moral. Vemos esto en palabras de La Taille:

La edad de 8 años es un "punto de inflexión" para muchas áreas del desarrollo psicológico. Esta es la época de las primeras conquistas de la reversibilidad operativa, por lo tanto de autonomía intelectual (Inhelder y Piaget, 1953). En términos de relaciones sociales, también es la era del comienzo de la coordinación mutua de acciones e intercambios verbal, lo que Mead (1934-1972) llamó teatro escénico, y Piaget ([1932] 1971), cooperación. (1995, p. 448).

Uno de los problemas que se presentan en el fragmento citado es que transfiere sin modificación la propuesta que Piaget utilizó para dar cuenta de la elaboración de conceptos lógico-matemáticos a la elaboración de nociones sociales. De esta manera la elaboración cognoscitiva del derecho al secreto es entendida como una construcción individual, dependiente de operaciones intelectuales, sin referencia al contexto social específico del que el sujeto participa y en el que toma conocimiento de sus derechos. El autor continúa diciendo:

En resumen, no es de extrañar que, en la génesis del secreto, encontremos la misma edad como marca el comienzo de la concepción moral de una nueva ley y, recordemos, un nuevo deber: guardar el secreto confiado por otros. Sin embargo, no es la edad de 8 años en sí mismo, lo cual es importante (la edad ciertamente puede variar según el culturas y experiencias personales de los sujetos), pero el hecho de que se pueda verificar un momento de evolución cuando nuevos instrumentos psicológicos y nuevos valores tienen lugar. Si nuestros análisis son correctos, la génesis del secreto se une a otras conquistas psicológicas, especialmente cognitivas y morales. (p. 448).

En el párrafo precedente, el autor desprende la construcción de conocimientos sociales de una progresión necesariamente vinculada a la edad, pero no logra independizarla del desarrollo intelectual general independientemente del contexto específico en el que se produce el conocimiento. Desde otras perspectivas que trabajaremos a continuación, el contexto de producción de conocimientos aporta mucho más que el tiempo en el que aparece una determinada noción que depende del desarrollo intelectual, funcionando como una restricción que posibilita determinada elaboración de ideas e inviabiliza otras.

Diferenciándose de estos estudios sobre la construcción conceptual de los espacios personales, encontramos una investigación realizada por Helman y Castorina $(2005,2007)$ sobre las ideas infantiles de los derechos en la escuela, entre ellos, el derecho a la intimidad. 
Los resultados de esta investigación muestran que todos los sujetos entrevistados (niños y niñas entre 8 y 12 años) reconocen el derecho a la intimidad considerando que es incorrecto que el docente intervenga sobre el espacio personal de los niños. Para la autora sólo es posible pensar una norma jurídica que regula, por ejemplo, los comportamientos tendientes a preservar la intimidad del niño, si primero se reconoce que existe un campo de actos de índole privada. La autora define al derecho a la intimidad como el reconocimiento de un conjunto de ámbitos personales susceptibles de ser pensados por el niño como derecho. Esta investigación también muestra que de manera generalizada los niños y niñas que reconocen el derecho a la intimidad lo entienden de manera condicionada. Lo que resulta crucial para entender esta perspectiva teórica es la índole de los condicionamientos. Estos son requisitos que, desde el punto de vista infantil, los niños deben cumplir para gozar de su derecho a la intimidad y están relacionados con algunos lineamientos de las prácticas escolares. De esta manera, encuentra que hay un reconocimiento de los derechos del buen alumno (HELMAN; CASTORINA, 2007), en tanto los sujetos de la muestra que reconocen el derecho a la intimidad entienden que sólo pueden gozar de él aquellos niños que no transgredan las normas escolares.

Esta investigación deja abierta la posibilidad de indagar y profundizar el estudio de las características de las ideas infantiles sobre el derecho a la intimidad. Esto es, ampliar la muestra a niños y niñas de menor edad, así como explorar si existen otras condiciones que los niños consideran que deben cumplir para hacerse acreedores del derecho en cuestión.

\section{Los problemas y objetivos del estudio}

Estudiar las ideas de los niños y las niñas sobre el derecho a la intimidad en la escuela es plantear la siguiente cuestión: por una parte, la escuela es uno de los contextos en los que el sujeto toma conocimiento de sus derechos infantiles; por otra parte, la institución educativa interviene en la vida personal de los niños y las niñas y promueve otras normas sociales que pueden llegar a contradecir la esfera íntima, como ser la construcción de un espacio público.

Está en curso una discusión relacionada con las atribuciones jurídicas de la Convención internacional sobre los derechos de los niños (ONU, 1989) que presenta las diferentes perspectivas que estuvieron en discusión cuando fue elaborada (ROSENBERG; SUSSEL MARIANO, 2010). Mientras que por un lado se promueven derechos relativos a la libertad individual, por otro, se establecen derechos que protegen a la infancia (como los referidos al trabajo infantil).

Los autores Rosemberg y Sussel Mariano (2010) sostienen que esta coexistencia de dos perspectivas en el interior de la Convención expresa los conflictos político-ideológicos presentes en las discusiones entre los representantes de los diferentes países que integraron los grupos de trabajo y la redacción de la misma. Según esta interpretación, la Convención es el producto de un acuerdo político entre las partes encontradas y por ello su texto presentaría ciertos conflictos entre esas diferentes versiones de la infancia. Para nosotros es indiscutible que los niños y las niñas participan en relaciones de dependencia y son claramente vulnerables. Esto debe ser considerado. De lo contrario, se corre el riesgo de que 
los niños y las niñas -con sus derechos de autonomía reconocidos- queden disociados de la necesaria construcción con los adultos de las condiciones que protegen sus derechos, debido a su responsabilidad de generar condiciones para el cumplimiento pleno de sus derechos.

Esta tensión se traslada al espacio escolar. A pesar de que la Convención Internacional sobre los Derechos de los Niños atribuye a la infancia el derecho a la privacidad, debe llamarse la atención sobre cierta dificultad de que este derecho se realice sin tensiones en el ámbito escolar.

Respecto del ámbito escolar, en palabras de Le Gal: "Solo con leer cierto número de principios centrales de la Convención, hacen pensar que es necesario crear otro sistema educativo para que la escuela se convierta en la escuela de los derechos del niño, de la libertad y de la ciudadanía participativa." (2005, p. 44).

El reconocimiento del derecho a la intimidad entra en contradicción con algunos aspectos y características de la escuela. En primer lugar, existe un conjunto de normas de la institución que puede tensionar el respeto a un espacio personal, por ejemplo, el hecho de que un docente tenga la responsabilidad de cuidar y proteger a los niños hace que intervenga fuertemente en la vida de ellos. En segundo lugar, no debemos olvidar que el reconocimiento de un espacio íntimo en los niños y las niñas, ajeno a la intervención por parte de la escuela, sería un límite a los aspectos disciplinadores y un límite a su vigilancia.

Esto nos llevó a elaborar las siguientes preguntas de investigación: ¿Cuáles son las ideas infantiles sobre la intimidad? ¿Qué características asumen estas ideas en la escuela? ¿Puede suponerse alguna relación entre prácticas escolares e ideas sobre el derecho a la intimidad? ¿Cuáles? ¿Cómo se visualiza en las ideas infantiles? ¿Puede hablarse de desarrollo de ideas sobre el derecho a la intimidad? ¿En qué términos?

Estas preguntas suponen el interés de elucidar las relaciones existentes entre elaboración conceptual infantil del derecho a la intimidad en la escuela y las prácticas escolares de las que participan los niños y las niñas.

\section{Objetivos específicos del estudio}

a) Identificar las ideas sobre el derecho a la intimidad en la escuela primaria en niños y niñas de contextos de pobreza urbana concurrentes a una escuela pública de la Ciudad Autónoma de Buenos Aires.

b) Establecer si es posible distinguir niveles de desarrollo conceptual en los argumentos de los niños y las niñas entrevistados/as.

c) Reconsiderar las restricciones institucionales sobre la formación de las ideas infantiles en el terreno moral.

\section{Tipo de estudio}

La investigación que aquí se presenta, se propone una indagación con un diseño transversal evolutivo de carácter cualitativo y exploratorio. 


\section{Muestra}

La muestra fue de 30 sujetos de 7 a 12 años de contextos de pobreza urbana (BATALLÁN; VARAS, 2002) que asisten a una escuela pública de CABA (Ciudad Autónoma de Buenos Aires), habitantes de asentamientos y barrios pobres del barrio de La Boca cuyos padres o tutores son asalariados pobres. Esta información fue suministrada por la directora del colegio. La selección de la muestra obedece a uno de los objetivos que estuvieron en el inicio de la investigación: comparar las ideas infantiles de niños de sectores medios, descriptas de manera general en estudios anteriores (HELMAN; CASTORINA, 2007, 2010; HORN; CASTORINA, 2010) con las que surgieran en esta indagación. Sin embargo, no se señalan posibles diferencias, ya que no aparecieron grandes distancias con las indagaciones anteriormente realizadas y resultó de mayor interés poder precisar las características de las ideas infantiles. Se seleccionó de modo intencional no probabilístico debido a que se usó un método de muestreo por cuotas (HERNÁNDEZ SAMPIERI; FERNÁNDEZ COLLADO; BAPTISTA LUCIO, 1991).

En la selección de niños y niñas se priorizó su pertenencia a un mismo grupo de clase. Por eso se agrupan niñas y niños por grado. El supuesto que está por detrás de este agrupamiento es que las prácticas escolares que se producen en un mismo grupo pueden tener incidencia en las ideas que ese grupo elabora sobre el derecho. Los sujetos que asisten a $2^{\circ}$ tienen entre 7 y 8 años, los que asisten a $4^{\circ}$ entre 9 y 10 y los que asisten a $6^{\circ}$ entre 11 y 12. Se presenta la distribución definitiva:

Tabela 1- distribución de niñas y niños

\begin{tabular}{c|cc:c}
\hline Grado & Niñas & Niños \\
\hline $2^{\circ}$ & 5 & 5 & 5 \\
\hline $4^{\circ}$ & 5 & $\vdots$ & 5 \\
\hline $6^{\circ}$ & & 5 & 5 \\
\hline
\end{tabular}

Fuente: Elaboración propia.

Las entrevistas contaron con el consentimiento informado de los entrevistados y las entrevistadas y la autorización de los referentes adultos responsables de ellos y ellas.

\section{Instrumentos para el relevamiento de la información}

Para llevar adelante los objetivos de la investigación se realizaron entrevistas individuales basada en los lineamientos del método clínico-crítico piagetiano (PIAGET, 1973; DELVAL, 2001). Este tipo de entrevistas permite reconstruir el punto de vista del niño y sus particularidades a partir de un diálogo con el sujeto que es guiado por las respuestas que este último va dando. En ellas se presentaban tres historias en las que se narraba una vulneración de un espacio personal por parte de una autoridad escolar. 
Transcribimos las tres historias presentadas a los sujetos de la muestra a partir de las que giraba la entrevista ${ }^{3}$ :

\begin{abstract}
Narrativa 1
Una niña está triste porque tiene un problema en la casa. La maestra, que sabe lo que a ella le está pasando, decide contarle esto al resto de sus compañeros de grado.

Narrativa 2 -Tomada de Helman (2007)

En el aula, en una hora de clases una nena escribe un papelito a una compañera y se lo pasa mientras la maestra está explicando. La maestra la descubre y le dice que le dé el papel. La nena no quiere darle el papelito con lo que escribió. Le dice a la señorita que le promete que lo va a guardar pero la señorita le vuelve a decir que se lo dé. Entonces la nena se lo entrega y la señorita se va a un costado del aula, abre el papel y lo lee, en silencio, para ella.
\end{abstract}

\title{
Narrativa 3
}

Juan sale del aula con permiso de la maestra. En ella quedó la maestra con el resto de los alumnos del grado. La maestra aprovecha esto para contarles a todos los chicos que Juan tiene muchos problemas de conducta y se porta muy mal. La maestra les pide a todos que lo ayuden.

Las entrevistas clínico-críticas suponen la actividad de entrevistador en dos direcciones: por un lado, la expectativa de que la voz infantil se exprese, de modo tal que sea posible su reconstrucción por parte del investigador (DUVEEN; GILLIGAN, 2013). Otorgar la voz a los niños y las niñas en las indagaciones sobre construcción de conocimiento infantil es una oportunidad de promover su derecho a ser escuchados (LEE, 2010). Por otro lado, está el lugar que se le otorga a la teoría en el momento de las actividades metodológicas de recolección de datos, que permite interpretarlos a medida que se van produciendo y repreguntar conforme a los interrogantes teóricos que guían las preguntas (CASTORINA; LENZI; FERNÁNDEZ, 1984; DELVAL, 2001).

\section{Análisis de los datos}

Para el análisis los datos obtenidos por medio de las entrevistas se utilizaron procedimientos cualitativos. Se realizaron, de esta manera, las categorías partiendo de recurrencias y convergencias en las respuestas dadas por los sujetos (DELVAL, 2001) que fueron interpretados a partir del marco teórico.

El análisis de las entrevistas clínico críticas implica la construcción de categorías que son validadas por otros y otras investigadoras. Esto se denomina acuerdo interjueces y tiene el objetivo de garantizar su validez. Se dio el material empírico a dos jueces expertos con un acuerdo del 95\%, lo que implica que en ese porcentaje hubo acuerdo con las categorías que se utilizaron para analizar la muestra.

3- Las entrevistas fueron realizadas en Ciudad de Buenos Aires, en el año 2009. 


\section{Las ideas infantiles sobre el derecho a la intimidad}

Como producto del análisis de los datos se elaboraron las siguientes categorías. Cabe señalar que los derechos infantiles, tal y como están sancionados en la Convención Internacional sobre los Derechos de los Niños, son entendidas por algunos juristas (LEIRAS, 1994) como una expectativa de trato deseable construida históricamente. Es decir, que un grupo social entiende y respalda simbólicamente cómo deberían ser tratados los sujetos, aunque esto sólo implique la dimensión del deber ser y no siempre sea consistente con lo que realmente sucede. Cuando estudiamos las ideas infantiles sobre algunos derechos, encontramos que los niños y las niñas tienen ciertas expectativas de trato deseable de los adultos y las adultas hacia ellos y ellas. Esta expectativa no siempre incluye el respeto a la intimidad. De esta manera, las categorías que aquí reseñamos muestran las diferentes expectativas de trato encontradas en la muestra.

\section{A. Expectativa de benefacción}

Las expectativas de benefacción son un conjunto de ideas infantiles en las que se reconoce que las acciones de las autoridades escolares deben estar orientadas a producir un bienestar en el niño y evitar que este sea dañado. El hecho de ser una expectativa de trato nos permite considerarla como el reconocimiento de cierta realidad jurídica (KOHEN, 2003). Sin embargo, al no haber estudiado las ideas infantiles sobre los derechos que pueden agruparse dentro de esta expectativa, no podemos describir la riqueza de ideas infantiles en torno a ellos, lo que sí podemos considerar es de qué manera este reconocimiento es prioritario o no respecto al derecho a la intimidad. En muchos casos, los niños y las niñas, ante la presentación de una narrativa en la que se vulneraba el derecho a la privacidad, entendían que la intromisión en la vida personal estaba habilitada por la intención benefactora de la maestra o los beneficios posteriores a la intervención.

Por ejemplo, encontramos como respuesta a la presentación de la Narrativa 1 en lo dicho por Oriana de $\left(7 ; 6^{4}\right)($ Entrevista 2, p. 3):

Entrevistador: Lo que hace la maestra ¿Qué te parece, Oriana?

Oriana: Está bien

Entrevistador: ¿Por qué?

Oriana: Porque después pueden ayudar con algo a esa nena.

$[\ldots]$

Entrevistador: ¿Por qué te parece que lo puede decir la maestra?

Oriana: Porque así la ayudan todos.

Aquí (lo que resulta relevante para nosotros), los sujetos no consideran la vulneración del derecho a la intimidad. La acción de la maestra es interpretada como una intervención legitima por ser benefactora. En estos casos, los sujetos parecen centrar su atención en la

4- Los números corresponden a la edad de los sujetos. El primer número corresponde los años y el segundo a los meses. En el caso indicado son 7 años y 6 meses. 
protección de los niños y las niñas y la evitación del daño en detrimento del respeto a las informaciones personales. No están sopesando derechos, actitud que podríamos atribuir a un niño o niña como jurista, tomando decisiones acerca de cuál es el derecho que le parece más relevante respetar. Las situaciones presentadas son interpretadas por una conceptualización infantil de expectativa de benefacción sin poder considerar el derecho a la intimidad implicado.

Para el sujeto no se constituye como observable la vulneración al derecho a la intimidad. En cambio, la situación es interpretada a la luz de otras expectativas de trato que, según Turiel (1984), se constituyen tempranamente, vinculadas con la evitación de un daño. Por lo tanto, estas ideas tienen relevancia en tanto pueden ser interpretadas como los primeros acercamientos a la elaboración conceptual de una expectativa de trato. Aunque ésta no incluya aún la consideración de un derecho por nosotros estudiado.

\section{B. Expectativa de respeto a la intimidad}

Ingresamos en los primeros reconocimientos de una expectativa de respeto a la intimidad. Esta puede encontrarse condicionada o incondicionada. Esto establece un quiebre con la expectativa de trato precedente donde no se reconocía como parte de ella el respeto al espacio personal de un niño o niña. Vemos un ejemplo (Entrevista 6, p. 4):

El entrevistador cuenta la narrativa 1 y pregunta el parecer del entrevistado sobre lo que hace la maestra.

Brandon (7, 11): Está mal.

Entrevistado: ¿Está mal? ¿Por qué está mal?

Brandon: Porque le contó a todos los chicos y la nena se puede enojar.

Entrevistado: ¿Y por qué se puede enojar?

Brandon: Porque no lo tiene que contar.

Entrevistado: ¿Por qué no?

Brandon: Porque es suyo...

Lo que resulta relevante de estos argumentos es que son los primeros reconocimientos de un espacio personal (LA TAILLE, 1996). Existe el reconocimiento jurídico de su respeto, expresado en una expectativa de trato que suceda. Los argumentos agrupados bajo esta categoría dan cuenta del reconocimiento que el/la docente no tenga libre acceso a la información personal de los alumnos.

\section{B.1. Alternancia de centramientos entre expectativa de respeto a la intimidad y expectativa de benefacción}

Hablamos de alternancia de centramiento cuando el niño o la niña pasan alternativamente por un reconocimiento del derecho a la intimidad y en otro momento, sobre una misma narrativa, dejan de considerar una expectativa de respeto a la intimidad para sólo considerar expectativas de benefacción. En estos casos, no hay condicionamiento porque no se conciben condiciones que limitan el derecho a la intimidad reconocido. 
Dependiendo del centramiento cognoscitivo del sujeto, reconoce el derecho a la intimidad u otra expectativa de trato. Un ejemplo (Entrevista 22, p. 7):

Entrevistador, luego de contar la primera narrativa del diseño, pregunta: ¿Y qué te parece lo que hizo la maestra?

Gonzalo (10; 2): Está bien.

Entrevistador: ¿Por qué?

Gonzalo: Porque cuando vos estás en clase no tenés que jugar, no tenés que correr, no tenés que pasarte papelitos, nada. Eso es para el recreo.

[...]

Entrevistador: Está bien. Te cuento: un nene de tu misma edad me dijo que por ahí no estaba muy bueno que la maestra lo agarrara y lo leyera porque era algo de las nenas y que no tenía por qué leer y meterse en las cosas de las nenas ¿Qué te parece lo que dice este nene?

Gonzalo: Está bien también.

Entrevistador: ¿Por qué está bien lo que dice?

Gonzalo: Porque no se tiene que meter con las cosas de las chicas

Entrevistador: ¿Y esto que me decías antes que te parece que estaba bien que lo leyera porque estaba en la hora de la maestra?

Gonzalo: Y sí, están bien los dos.

\section{B. 2. Expectativa de respeto a la intimidad condicionada}

Esta clasificación implica un primer nivel de distinción vinculado con el objeto de nuestra indagación. Sin embargo, este primer reconocimiento supone que la expectativa reconocida puede estar sujeta a diferentes condiciones. Bajo estas categorías encontramos, por una parte, que el niño tiene una expectativa de trato que supone que no deben acceder a su información personal. Sin embargo, ese límite al acceso de su espacio personal está sujeto a ciertas condiciones que el niño, la niña o la situación deben cumplir.

Existen diferentes condiciones que los sujetos consideran que deben cumplirse para que pueda gozarse del derecho a la intimidad, por ese motivo agrupamos estos condicionamientos en tres subcategorías. Sin embargo, no debe entenderse que estas subcategorías se excluyen unas a otras. Para un mismo sujeto el derecho a la intimidad puede estar limitado por más de una condición que detallaremos en las subcategorías. Es relevante, desde el punto de vista cognoscitivo, la distinción de una expectativa de respeto a la intimidad y ciertas condiciones que la limitan (Entrevista 13, p. 9).

Entrevistador presenta la Narrativa 2 y pregunta: Y esto que la maestra lo agarre y lo lea, ¿Qué te parece?

Jazmín (11; 02): Por un lado no me parece justo porque pueden estar contando algo personal que no lo quieren que sepa nadie y la señorita lo agarra...

Entrevistador: ¿Y entonces? ¿Decías por un lado me parece injusto, te parece injusto que lo haga?

Jazmín: Y sí, la señorita injusto, porque tampoco tiene la razón de agarrar ese papel y leerlo sin que ella sepa de lo que es. 
Entrevistador: ¿Qué te parece que tendría que hacer la maestra?

Jazmín: Eh... Llamar a las dos y hablarles.

Entrevistador: ¿Y puede leerlo el papel?

Jazmín: No.

Hasta aquí hay un reconocimiento de una expectativa de respeto al espacio personal, veamos cómo continúa la entrevista (Entrevista 13, p. 9).

Entrevistador: Ahora, suponete que la nena es una nena que se portaba mal, siempre pasaba papelitos en clase no prestaba atención en clase y pasaba todo el tiempo papelitos en clase. Y la primera vez la maestra no lo leyó, la segunda tampoco, pero en esta tercera ya la maestra lo agarra y lo lee. ¿Qué te parece lo que hace esta maestra?

Jazmín: Me parece que es porque si la nena molesta a los demás y los demás quieren escuchar. Me parece que la señorita tiene que agarrar ese papel y leerlo a ver de qué se trata que están todos los días pasándose papeles.

Sin embargo, el reconocimiento de una expectativa de respeto a la intimidad está condicionada por el comportamiento escolar de la niña.

Las subcategorías que condicionan el derecho a la intimidad son las que presentamos a continuación.

La primera es la que hemos denominados condicionado por resultado de las acciones de la maestra. Dentro de esta subcategoría subsumimos aquellas ideas infantiles en las que los niños y las niñas registran un espacio personal y una expectativa de respeto. Parece, sin embargo, ser más fundamental el resultado benefactor o la evitación de un daño que puede tener la intervención de la autoridad escolar sobre el espacio personal del niño o la niña.

La segunda es el condicionado por intención benefactora de la maestra. Esta subcategoría tiene cierto parecido con la anterior: en este caso, lo que limita el derecho a la intimidad es cierta benefacción en las intenciones de la maestra. Sin embargo, lo que la diferencia es que lo que habilita a la maestra a intervenir en el espacio personal de los niños y las niñas es la intención de producir un efecto positivo a la niña a quien se vulnera su espacio personal.

La tercera es condicionado por acciones anti-normativas del alumno. Dentro de esta, subsumimos el límite al derecho a la intimidad reconocido en aquellas situaciones en las que los alumnos o alumnas no se comportan acorde a las expectativas disciplinarias de la escuela y buen desempeño escolar. De todas maneras, no cabe establecer una diferenciación tajante con los condicionamientos anteriores. Esto legitima que, desde la mirada infantil, la intromisión en los aspectos personales de los niños es cumplimiento de un fin benefactor implicado en el restablecimiento del orden escolar.

\section{B.3 Expectativa de respeto a la intimidad incondicionada}

Decimos que el derecho a la intimidad se encuentra incondicionado cuando el respeto por el espacio personal e íntimo del niño o la niña no está sujeto a otra condición que la de ser niño o niña. El referente empírico de esto es que el sujeto entrevistado considere a lo 
largo del interrogatorio clínico, alrededor de una misma narrativa, que no debe vulnerarse el espacio personal en ninguna de las circunstancias planteadas (como por ejemplo, que la niña que pasa el papelito tiene malas notas o lo hace en reiteradas oportunidades). Es importante remarcar que ninguno de los sujetos de la muestra sostuvo la incondicionalidad en todas las narrativas presentadas y sólo cuatro la sostuvieron en las consideraciones de alguna de las situaciones planteadas. Uno de los ejemplos (Entrevista 19, p. 3):

El entrevistador cuenta la primera narrativa del diseño y pregunta: Bueno, ¿qué te parece la historia que te conté?

Fabián (11; 7): Si tiene problemas familiares, la nena, la maestra no le tiene que contar al resto, porque es un problema familiar de una sola persona.

Entrevistador: Está bien. Suponé que la maestra lo cuenta a todos los compañeros y los compañeros la ayudan, no se siente avergonzada, sino que la ayudan, ¿no?, y ella se siente mejor. ¿Qué te parece en ese caso lo que hizo la maestra?

Fabián: Está bien. [...] Porque... ahí es como pedirle ayuda a los compañeros, por ahí le hablan los compañeros, así a ayudarla a animarla y que por ahí, esos problemas familiares la nena, como por ahí se siente... si es un problema así, grave en la familia, se va a sentir como... avergonzada. Entrevistador: Está bien. ¿Pero está bien lo que hace la maestra o está bien lo que hacen los chicos? Fabián: Está bien lo que hacen los chicos. [...] -Lo que hace la maestra...- Está así, medio más o menos bien, pero no le tiene que contar lo que pasó a chicos así.

En este caso el niño considera la incondicionalidad del derecho a la intimidad, es decir que por más que la maestra ayude a la niña con su intervención, esto no anula la existencia del derecho a la intimidad.

En este recorrido por las ideas infantiles, encontramos un soporte para nuestra hipótesis de que estas nociones están canalizadas, posibilitadas y limitadas por la participación de los niños y las niñas en las prácticas escolares de la que recorta su objeto. Las respuestas infantiles mostraron que el derecho a la intimidad en la escuela aparece limitado por algunas características que son propias de las prácticas escolares. Esto se da en las ideas que hemos denominado expectativa de respeto a la intimidad condicionada, donde el derecho a la intimidad reconocido encuentra su límite en la intensión benefactora de la maestra o por el desempeño escolar del alumno o la alumna. En otras palabras, los datos sugieren que la elaboración misma de las nociones infantiles sobre un derecho determinado incluye aspectos del contexto específico en el que se elabora: el derecho se suspende, desde la perspectiva infantil, cuando el beneficiario contradice normas de esa institución en particular.

\section{Discusión}

Hemos mostrado los grandes hitos en las ideas infantiles encontrados, desde el no reconocimiento de una expectativa de respeto a la intimidad a su reconocimiento episódicamente incondicionado. Subyace a esta presentación una discusión en torno al concepto de desarrollo de ideas (CHAPMAN, 1998). 
Los datos arrojan algunas pistas para entender en qué términos se da la progresión conceptual que hemos detallado arriba. Presentaremos un gráfico en el que se muestra la frecuencia de cada uno los grupos de ideas descriptos en su relación con la edad por edad y por narrativa:

Gráfico 1- Distribuición de las categorias diferenciando narrativas y grado al que pertenecen los sujetos

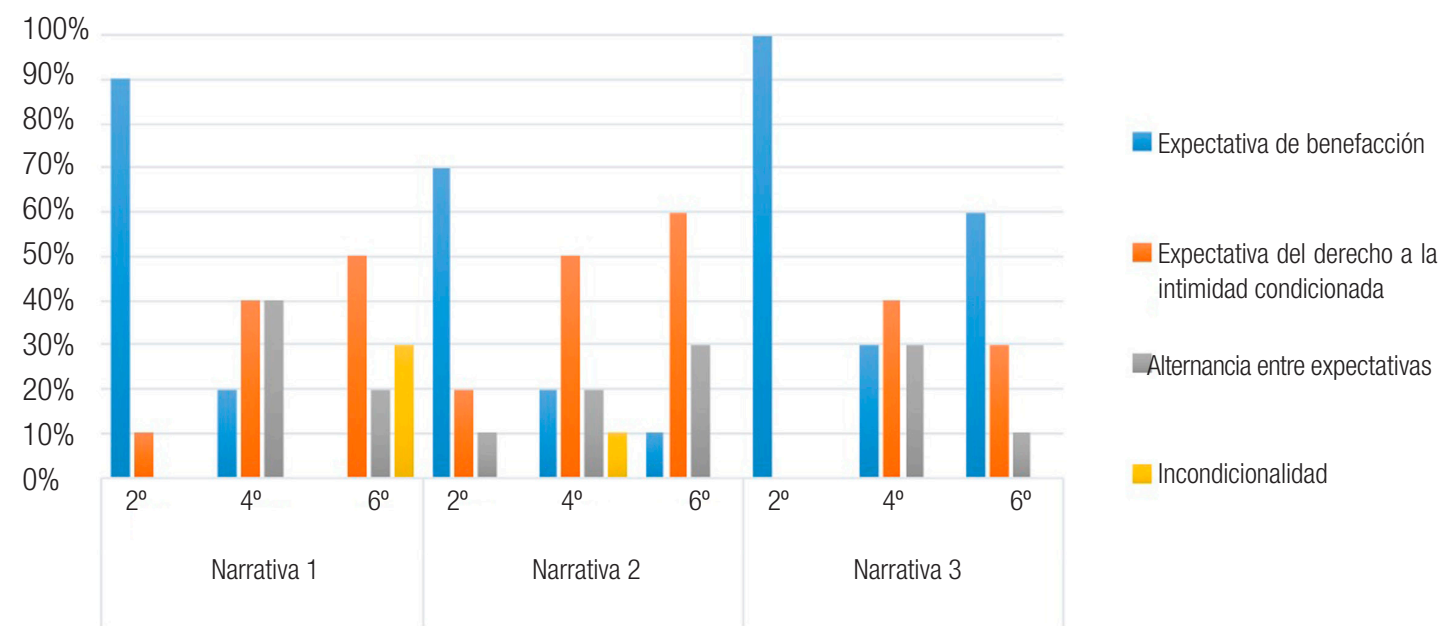

El gráfico muestra cómo se distribuye cada categoría por grupo de edad diferenciando por narrativa. Por ejemplo, del grupo de $2^{\circ}$ la Narrativa 1 fue explicada desde una expectativa de benefacción por el $90 \%$ y solo el $10 \%$ por una expectativa de derecho a la intimidad condicionada. Ese mismo grupo de edades -los del $2^{\circ}$-, distribuye sus respuestas de otra manera ante la Narrativa 2, donde el $70 \%$ la explica desde una expectativa de benefacción.

Fuente: Elaboración propia.

En primer lugar, no hay dependencia estricta con la edad, sino que es más bien un desarrollo conceptual. El no reconocimiento de una expectativa de respeto a la intimidad se agrupa en los sujetos más pequeños, el reconocimiento de esta expectativa de respeto a la intimidad se distribuye en todo el rango de edades. Esto nos coloca nuevamente en diálogo con los trabajos de La Taille, Bedoia y Giménez (1991) y La Taille (1998). Sus estudios hablan de una fuerte correspondencia entre consideraciones conceptuales y edad. Nuestros resultados ponen en cuestión esa aseveración.

En segundo lugar, la construcción de una noción incondicionada no implica un cambio general de las consideraciones infantiles, sino un reconocimiento parcial de ella. Los niños y las niñas que identificaron la incondicionalidad del derecho sólo lo hacían en una de las situaciones o narrativas planteadas y no en todas. Esta elaboración conceptual lejos de ser un punto de llegada final del pensamiento infantil, está sujeto a las diferentes situaciones que el sujeto debe considerar.

Nuestros resultados permiten pensar en una versión diferente de progresión cognoscitiva en nociones sociales a las que subyacen las otras investigaciones que venimos 
comentando -La Taille, Bedoia y Giménez (1991) y La Taille (1998; BATAGLIA; FERREIRA; SALADINI, 2020)-, que la hacían depender exclusivamente de la actividad intelectual individual. Se reconoce la existencia de ideas menos objetivas y otras más objetivas. Alcanzar estas últimas no es un hecho al que todos los sujetos lleguen, ni lo hacen a la misma edad. En este sentido, es más que elocuente a este respecto Emilia Ferreiro:

No se llega a las operaciones concretas como se llega a tener seis años o a sentarse en un banco de primer año. Se llega después de múltiples conflictos, de compensaciones parciales, de intentos fallidos por resolver problemas. No se llega por un milagroso proceso de maduración que nos llevaría tranquilamente de un estadio al siguiente. (1999, p. 9).

Nosotros podemos agregar que por más esfuerzo intelectual que haga el niño o la niña, no todos los sujetos llegan a las conceptualizaciones más avanzadas ya que no es una cuestión meramente intelectual. Una vez que se alcanzan estas ideas más objetivas del derecho a la intimidad, no implica que el sujeto pueda considerar todas las situaciones desde este punto de vista. Un mismo sujeto sostiene versiones menos objetivas del derecho a la intimidad en otras situaciones.

¿Qué posibles hipótesis nos explican estas particularidades del pensamiento infantil? Para interpretar estos resultados hemos elaborado algunas hipótesis provisorias que nos invitan a continuar con otras investigaciones para poder sostenerlas con mayor validez. Los datos parecen mostrar que las construcciones que hacen los niños y las niñas acerca de su derecho a la intimidad no se dirigen inevitablemente a la incondicionalidad, siendo ésta sólo reconocida en pocos casos. Desde nuestra perspectiva, que los niños y las niñas consideren que este derecho está limitado por las acciones benefactoras de la maestra puede ser una expresión de que la situación escolar pone condiciones a su elaboración conceptual.

Esto no debe pensarse como si la elaboración conceptual y las condiciones o prácticas sociales fueran externas y se limitan a favorecer o impulsar el pensamiento, más bien podría hablarse de que las prácticas escolares serían las condiciones constitutivas de la elaboración conceptual. En este punto, nos diferenciamos de la perspectiva de La Taille (1995, 1996, 1998), donde el desarrollo era explicado por una actividad intelectual que empujaba a los niños a ideas más objetivas sobre el derecho a la intimidad.

El estudio presentado sugiere que la construcción de conocimientos tiene lugar en las prácticas sociales mismas y es dentro de ellas que el sujeto recorta el derecho a la intimidad. Dentro de lo que denominamos prácticas sociales, incluimos las acciones institucionales directamente restrictivas al espacio personal, como pueden ser las sanciones, la vigilancia y las asimetrías entre niños y docentes. A su vez, un conjunto de normas escolares que van más allá de las acciones puramente disciplinadoras, como puede ser el respetar el bien común por sobre los aspectos individuales, o las características benefactoras de los agentes institucionales. También debe considerarse el desarrollo histórico que produjo un espacio personal y privado sin el cual sería impensable elaboraciones infantiles sobre la intimidad y su derecho. De esta manera, las restricciones al conocimiento surgen con las complejas relaciones institucionales 
que incluyen en una misma totalidad institucional relaciones de poder e interjuego de normas y suponen una historia de formación de las nociones.

No somos los primeros en encontrar que las ideas infantiles suelen estar sujetas a cierta expectativa benefactora, también lo señala, por ejemplo, Barreiro $(2012,2018)$, además de Castorina, Lenzi y Fernández (2000), Aisenberg y Kohen (2000), Lenzi y otros autores (2005), que estudiaron sobre ideas políticas.

Si en la Convención internacional sobre los derechos del niño (ONU, 1989) el reconocimiento de derechos incluye, a su vez, el cuidado de la integridad de las personas (el cuidado a su alimentación, vivienda digna, evitación del maltrato infantil) y la promoción de cierta ciudadanía infantil, desde la perspectiva infantil lo que parece regular el derecho en las escuelas son derechos como los primeros. Es decir, el niño reconoce muy tempranamente que la escuela debe protegerlo y no maltratarlo, tanto es así que en las justificaciones infantiles tienen mayor peso la evitación de un daño personal, como puede ser la tristeza provocada por un suceso privado, que el respeto a la intimidad. Estas consideraciones infantiles son preponderantes y suponen en muchos casos el desconocimiento de una expectativa de respeto a la intimidad.

\section{Conclusión}

A lo largo del artículo pudimos plantear que los niños y las niñas poseen diferentes ideas y que en algunas de ellas se distinguen con mayor claridad el reconocimiento de una expectativa de respeto a la intimidad. Esto parece abonar la idea de que puede hablarse de un desarrollo intelectual de la noción. Sin embargo, también se expusieron resultados en los que se vio que no todos los sujetos llegaban a lo que hemos considerado niveles más avanzados u objetivos de la noción, e incluso los pocos sujetos que lo hacían no lo sostenían en todas las situaciones que se presentaban. Hay un avance en las concepciones infantiles, este avance implica la reconstrucción de las nociones sociales tal y cómo aparecen en la vida colectiva y no como una abstracción de la noción en tanto contenido externo a las prácticas sociales. Esto sugiere que la construcción de ideas que realizan se produce a partir su experiencia social. Puede sostener que en la elaboración de nociones sociales asistimos a un caso de producción cognoscitiva que implica considerar la actividad social de los niños y las niñas mientras construyen sus ideas.

Esto último supone una particular relación epistémica entre este tipo de objetos de conocimiento y la actividad constructiva del individuo. Piaget (1971) concebía a la relación epistémica como una relación dialéctica entre el sujeto y el objeto. La relación del sujeto con los objetos de conocimiento social tiene particularidades que se desprenden de los resultados de investigación. En primer lugar, estos objetos de conocimiento, para ser reconocidos por el sujeto deben haber sido construidos socio-históricamente. En segundo lugar, en tanto objeto social, el sujeto lo recorta de las mismas prácticas sociales que lo contornean. Es decir, si encontramos que el derecho a la intimidad para la mayoría de los niños y las niñas se encuentra condicionado, es porque las prácticas sociales en las que ellos y ellas conocen este derecho es poco reconocido, o está supeditado a instancias de control institucional. Esto insinúa que los niños y las niñas no podrían elaborar ideas 
sobre su derecho a la intimidad si occidente no hubiera elaborado históricamente un espacio personal o privado. En otras palabras, la construcción histórica del ese espacio es el punto de partida para que la teoría del derecho a declare un derecho la intimidad para los niños y las niñas. A su vez, ese derecho en la vida escolar asume características propias que no son idénticas a la declaración de la Convención internacional sobre los derechos de los niños. Los niños y las niñas toman conocimientos sobre sus derechos en la vida social misma y por ello sus ideas expresan las características del derecho en la escuela y no el deber ser jurídico. De esta forma puede hablarse de una radical dialéctica entre el individuo y la sociedad en la construcción de conocimiento social, donde todo avance cognoscitivo individual supone su inescindible relación con las prácticas sociales en las que se produce el conocimiento.

Por último, es importante señalar que los resultados planteados sugieren fuertemente que las prácticas escolares tienen efectos en las elaboraciones cognoscitivas de los niños y las niñas acerca de su derecho a la intimidad. Esto permite suponer que la participación en prácticas escolares respetuosas de los derechos infantiles podría promover otras consideraciones de los/as alumnos/as acerca de los mismos. Se enfatiza de esta manera las potencialidades de la escuela en tanto institución pública, para formar políticamente a los alumnos como ciudadanos. Se pueden generar, así, situaciones reguladas por el reconocimiento de los derechos infantiles que habilitan en los niños y las niñas la posibilidad de pensar sobre sus derechos, reconocerlos y exigir que sean respetados.

\section{Referencias}

AISENBERG, Beatriz; KOHEN, Raquel. Las hipótesis presidencialistas infantiles en la asimilación de contenidos escolares sobre el gobierno nacional. In: CASTORINA, José Antonio; LENZI, Alicia (comp.). La formación de los conocimientos sociales en los niños. Barcelona: Gedisa, 2000. p. 181-200.

ALTMAN, Irwin. Privacy as an interpersonal boundary process. In: VON CRANACH, Mario et al. (ed.). Human ethology: claims and limits as a new discipline. Cambridge: Cambridge University Press, 1979. p. 95-132.

BARREIR0, Alicia. El desarrollo de las justificaciones del castigo: ¿conceptualización individual o apropiación de conocimientos colectivos? Estudios de Psicología, Madrid, v. 33, n. 1, p. 67-77, 2012.

BARREIR0, Alicia. El proceso de ontogénesis de las representaciones sociales de la justicia en niños y adolescentes. In: BARREIR0, Alicia (comp.). Representaciones sociales, prejuicio y relaciones con los otros: la construcción del conocimiento social y moral. Buenos Aires: Unipe, 2018. p. 129-154.

BATAGLIA, Patrícia U. R.; FERREIRA, Rafael R.; SALADINI, Ana Claudia. Entrevista prof. Dr. Yves de la Taille. Schème, Marília, v. 12, n. 1, p. 232-252, 2020. Disponible en: http://revistas.marilia.unesp.br/index.php/ scheme/article/view/10754. Acceso en: mayo 2020.

BATALLÁN, Graciela; VARAS, Rene. Regalones, maldadosos, hiperkinéticos: la educación de niños y niñas de 4 años que viven en pobreza urbana. Santiago de Chile: LOM, 2002. 
CASTORINA, José Antonio. Psicología genética y psicología social: ¿dos caras de una misma disciplina 0 dos programas de investigaciones compatibles? In: BARREIRO, Alicia (comp.). Representaciones sociales, prejuicio y relaciones con los otros: la construcción del conocimiento social y moral. Buenos Aires: Unipe, 2018. p. 33-54.

CASTORINA, José Antonio; FAIGENBAUM, Gustavo. Restricciones y conocimiento de dominio: hacia una diversidad de enfoques. In: CASTORINA, José Antonio; LENZI, Alicia (comp.). La formación de los conocimientos sociales en los niños: investigaciones psicológicas y perspectivas educativas. Barcelona: Gedisa, 2000. p. 155-177.

CASTORINA, José, Antonio; LENZI, Alicia; FERNÁNDEZ, Alicia. Algunas reflexiones sobre una investigación psicogenética en conocimientos sociales: la noción de autoridad escolar. In: CASTORINA, José Antonio; LENZI, Alicia (comp.) La formación de los conocimientos sociales en los niños. Barcelona: Gedisa, 2000. p. 41-58.

CASTORINA, José, Antonio; LENZI, Alicia; FERNÁNDEZ, Susana. Alcances del método de exploración crítica en psicología genética. In: CASTORINA, José, Antonio et al. (ed.). Psicología genética. Buenos Aires: Miño y Dávila, 1984. p. 83-118.

CHAPMAN, Michael. Contextualidad y direccionalidad del desarrollo cognitivo. Human Development, California, v. 31, p. 92-106, 1998.

DELVAL, Juan. Descubrir el pensamiento de los niños. Introducción a la práctica del método clínico. Barcelona: Paidós, 2001.

DUVEEN, Gerard; GILLIAN, Carol. On interviews: a conversation with Carol Gilligian. In: JOVCHELOVITCH; Sandrea; WAGONER, Brady (ed.). Development as social process. London: Routledge, 2013. p. 124-132.

FERREYRA, Julián; HORN, Axel; CASTORINA, José Antonio. Las ideas infantiles sobre el derecho a la intimidad: sus particularidades desde el medio virtual. Revista Investigaciones en Psicología, Buenos Aires, v. 17, n. 3, p. 25-43, 2012.

FERREIRO, Emilia. Vigencia de Jean Piaget. México, DC: Siglo XXI, 1999.

HELMAN, Mariela. Los derechos en el contexto escolar: relaciones entre ideas infantiles y prácticas educativas. In: CASTORINA, José Antonio (coord.). Desarrollo del conocimiento social: prácticas, discursos y teoría. Buenos Aires: Miño y Dávila, 2010. p. 215-235.

HELMAN, Mariela; CASTORINA, José Antonio. La institución escolar y las ideas de los niños sobre sus derechos. In: CASTORINA, José Antonio (ed.). Cultura y conocimientos sociales: desafíos a la psicología del desarrollo. Buenos Aires: Aiqué, 2007. p 219-242.

HERNÁNDEZ SAMPIERI, Roberto; FERNÁNDEZ CALLADO, Carlos; BAPTISTA LUCIO, Pilar. Metodología de la Investigación. México, DC: MacGraw-Hill, 1991. 
HIRSCHFEL, Lawrence. ¿La adquisición de categorías sociales se basa en una competencia dominioespecífica 0 en transferencias de conocimientos? In: HIRSCHFEL, Lawrence; GELMAN, Susan (ed.). Cartografía de la mente: la especificidad de dominio en la cognición y en la cultura: orígenes, procesos y conceptos. v. 1. Barcelona: Gedisa, 2002. p. 285-328.

HORN, Axel; CASTORINA, José Antonio. Las ideas infantiles sobre la privacidad: una construcción conceptual en contextos institucionales. In: CASTORINA, José Antonio (coord.). Desarrollo del conocimiento social: prácticas, discursos y teoría. Buenos Aires: Miño y Dávila, 2010. p. 191-214.

KARMILOF-SMITH, Annette. Más allá de la modularidad de la mente. Madrid: Alianza, 1994.

KOHEN, Raquel. La construcción infantil de la realidad jurídica. 2003. Tesis (Doctorado) - Facultad de Psicología, Universidad Autónoma de Madrid, Madrid, 2003.

LA TAILLE, Yves de. A gênese da noção de segredo na criança. Psicología, Brasilia, DF, v. 12, n. 3, p. 245-251, 1996.

LA TAILLE, Yves de. La genèse de la conception du droit au secret chez l'enfant. Enfance, Montpellier, v. 48, n. 4, p. 443-450, 1995.

LA TAlLLE, Yves de. Limites: três dimensões educacionais. São Paulo: Ática, 1998.

LA TAILLE, Yves de; BEDOIA, Graziela; GIMENEZ, Patricia. A construção da fronteira moral da intimidade: o lugar da confissão na hierarquia de valores morais em sujeitos de 6 a 14 años. Psicologia, Brasilia, DF, v. 7, n. 2, p. 91-110, 1991.

LE GAL, Jean. Los derechos del niño en la escuela: una educación para la ciudadanía. Barcelona: Graó, 2005.

LEE, Nick. Vozes das crianças, tomada de decisão e mudança. In: MÜLLER, Fernanda (org.). Infância em perspectiva: políticas, pesquisas e instituições. São Paulo: Cortez, 2010. p. 42-64.

LEIRAS, Marcelo. Los derechos del niño en la escuela. Buenos Aires: UNESCO, 1994.

LENZI, Alicia et al. La construcción de conocimientos políticos en niños y adolescentes. Un desafío para la educación ciudadana. In: CASTORINA, José Antonio (coord.). Construcción conceptual y representaciones sociales: el conocimiento de la sociedad. Buenos Aires: Miño y Dávila, 2005. p. 71-97.

ONU - Organización de la Naciones Unidas. Convención sobre los Derechos del Niño. [S. I.]: ONU, 1989. Asamblea general.

PIAGET, Jean. El criterio moral en el niño. Barcelona: Fontanella, [1932] 1971.

PIAGET, Jean. La representación del mundo en el niño. Madrid: Morata, 1973. 
PIAGET, Jean. Psicología y epistemología. Barcelona: Ariel, 1971.

ROSEMBERG, Fúlvia; MARIANO, Carmen Lucia. A Convenção Internacional sobre os Direitos da Criança: debates e tensões. Cadernos de Pesquisa, São Paulo, v. 40, n. 141, p. 693-728, dez. 2010.

TURIEL, Elliot. El desarrollo del conocimiento social. Moralidad y convención. Madrid: Debate, 1984.

Recibido en: 08.05.2020

Revisado en: 21.07 .2020

Aprobado en: 29.09.2020

Axel César Horn es doctor en Educación de la Facultad de Filosofía y Letras de la Universidad de Buenos Aires y magíster en Pedagogías Críticas y Problemáticas Socioeducativas. Es profesor adjunto de Psicología Genética en la Facultad de Psicología de la Universidad Nacional de La Plata. 